\title{
Comparison of Bleeding Profiles of Sugammadex and Neostigmine in Orthotopic Liver Transplantation
}

\author{
Hong Liang ${ }^{1}$, Launia White ${ }^{2}$, Ryan M Chadha ${ }^{2}$, and J Ross Renew ${ }^{1}$ \\ ${ }^{1}$ Mayo Clinic Hospital Jacksonville \\ ${ }^{2}$ Mayo Clinic Florida
}

October 22, 2021

\begin{abstract}
In the era of "fast-track' liver transplantation (LT), neuromuscular blockade (NMB) antagonists such as neostigmine or sugammadex are used to achieve the restoration of neuromuscular function. While sugammadex reverses NMB faster than neostigmine, it has been shown to prolong prothrombin time (PT) and activated thromboplastin time (aPTT). However, this agent's impact on coagulation during LT is not understood. We compare bleeding risk associated with sugammadex versus neostigmine during liver transplantation. This is a single-center, retrospective review of LT patients who received NMB antagonists intraoperatively between 01/01/2015 to 05/31/2018 at Mayo Clinic in Florida. The primary outcomes were postoperative day (POD) 0-1 bleeding events and POD 0 values of aPTT and INR. Total 241 patients were included, with 135 patients in the neostigmine group (NG) and 106 in the sugammadex group (SG). POD 0-1 postoperative bleeding requiring transfusion occurred in $20 \%$ of NG versus $10.4 \%$ in SG. POD 0-1 re-operation for bleeding occurred in $1.5 \%$ in NG vs. $0 \%$ in SG. POD 0 mean INR was $2.0 \pm 0.4$ in both groups. POD 0 mean aPTT was $45.5 \pm 7.9$ in NG vs. $49.3 \pm 9.0$ in SG. Our retrospective study suggests that sugammadex is not associated with an increased risk of bleeding compared to neostigmine use.
\end{abstract}

\section{Title Page}

Title :

Comparison of Bleeding Profiles of Sugammadex and Neostigmine in Orthotopic Liver Transplantation

Running title :

Sugammadex vs. neostigmine in liver transplant

Authors :

Hong Liang, DO ${ }^{1}$, Ryan Chadha ${ }^{1}$, MD, Launia J White ${ }^{2}$, J. Ross Renew MD $^{1}$ FASA FASE

Department of Anesthesiology and Perioperative Medicine, Mayo Clinic Florida

Department of Health Sciences Research, Mayo Clinic Florida

\section{Address correspondence to:}

Corresponding author: Hong Liang, DO

Email: liang.hong@mayo.edu

Address: Mayo Clinic Florida, 4500 San Pablo Road, Jacksonville, FL 32224

Phone: +1 (904) 9563328

Fax: +1 (904) 956-3332 


\section{All authors disclosure :}

1. No funding source supports the submitted work.

2. JRR has completed Merck-funded research. Funds to employer. HL and RC have nothing to disclose

3. No other associations.

Abstract

Comparison of Bleeding Profiles of Sugammadex and Neostigmine in Orthotopic Liver Transplantation

H Liang, DO, R Chadha, MD, L White, J.R Renew, MD FASA FASE

WHAT IS ALREADY KNOWN ABOUT THIS SUBJECT :

Sugammadex is known to prolong prothrombin time (PT) and activated thromboplastin time (aPTT); however, there are still controversies about the relation between sugammadex and bleeding. Liver transplant recipients often present with abnormal coagulation profiles and other comorbidities before liver transplantation and have a higher risk of postoperative bleeding. We compare bleeding risk associated with sugammadex versus neostigmine during liver transplantation.

WHAT THIS STUDY ADDS :

Our retrospective study results demonstrate that sugammadex is not associated with increased postoperative bleeding events, had less PONV, did not affect the organ rejection rate, and did not affect hospital length of stay. We provide incremental evidence sugammadex can be considered as an alternative to antagonize neuromuscular blockade following orthotopic liver transplantation.

\section{Introduction}

In the era of "fast-track' liver transplantation (LT), neuromuscular blockade (NMB) antagonists such as neostigmine or sugammadex are used to achieve the restoration of neuromuscular function. While sugammadex reverses NMB faster than neostigmine, it has been shown to prolong prothrombin time (PT) and activated thromboplastin time (aPTT). However, this agent's impact on coagulation during LT is not understood. We compare bleeding risk associated with sugammadex versus neostigmine during liver transplantation.

\section{Methods}

This is a single-center, retrospective review of LT patients who received NMB antagonists intraoperatively between $01 / 01 / 2015$ to $05 / 31 / 2018$ at Mayo Clinic in Florida. The primary outcomes were postoperative day (POD) 0-1 bleeding events and POD 0 values of aPTT and INR.

\section{Results}

Total 241 patients were included, with 135 patients in the neostigmine group (NG) and 106 in the sugammadex group (SG). POD 0-1 postoperative bleeding requiring transfusion occurred in $20 \%$ of NG versus $10.4 \%$ in SG. POD 0-1 re-operation for bleeding occurred in $1.5 \%$ in NG vs. $0 \%$ in SG. POD 0 mean INR was $2.0 \pm 0.4$ in both groups. POD 0 mean aPTT was $45.5 \pm 7.9$ in NG vs. $49.3 \pm 9.0$ in SG.

\section{Conclusions}

Our retrospective study suggests that sugammadex is not associated with an increased risk of bleeding compared to neostigmine use.

Key words : Sugammadex, liver transplantation, neuromuscular blockade, hemorrhage

Name of the Author to whom requests for offprints should be sent :

Hong Liang DO

Address: Mayo Clinic Florida, 4500 San Pablo Road, Jacksonville, FL 32224 


\section{Text}

\section{Introduction}

Liver transplantation is the second common transplant surgery in the United States ${ }^{1}$, and it remains the first-line treatment for patients with end-stage liver disease. With advances in surgical technique, anesthetic management, multidisciplinary patient care, and immunosuppression, the procedure has a greater than $90 \%$ 1-year survival and greater than $75 \% 5$-year survival. ${ }^{1}$ Due to this multidisciplinary approach, numerous centers have been able to perform the procedure with fewer perioperative complications allowing for extubation in the operating room and transfer to the floor, bypassing the intensive care unit (ICU), a process known as "fast-tracking."

Traditionally, anticholinesterase inhibitors have been used as a neuromuscular blockade antagonist (NMBA) prior to extubation for patients undergoing procedures requiring neuromuscular blockade. Over the last decade, a novel neuromuscular blockade antagonist, sugammadex, has become available as an alternative for reversing aminosteroidal neuromuscular blocking agents. ${ }^{2}$ Sugammadex is a modified cyclodextrin that selectively encapsulates and reverses the effects of steroidal neuromuscular relaxants. Compared to neostigmine, sugammadex has a lower risk of adverse events such as bradycardia and a lower incidence of postoperative nausea and vomiting (PONV). ${ }^{3}$ However, sugammadex is known to prolong prothrombin time (PT) and activated thromboplastin time (aPTT) ${ }^{4}$ This characteristic is of particular concern during liver transplantation as coagulopathy is common, and postoperative hemorrhage requiring re-operation can occur in approximately $10-15 \%$ of patients. ${ }^{5}$

Compared to the general population, liver transplant recipients often present with abnormal coagulation profiles and other comorbidities prior to liver transplantation and have a higher risk of postoperative bleeding. Data on the impact of sugammadex on coagulation in orthotopic liver transplantation surgery patients is limited. The primary goal of this study was to compare the bleeding risk of sugammadex versus neostigmine, with secondary outcomes focused on the incidence of organ rejection, PONV, and length of hospital stay (LOS) in liver transplant recipients. We hypothesize that the use of sugammadex has no impact on postoperative bleeding, organ rejection, and LOS, and may reduce the incidence of PONV when compared to neostigmine.

\section{Methods}

This study was conducted under Mayo Clinic IRB 19-005899. It is a single-center, retrospective review of patients who underwent orthotopic liver transplantation between 01/01/2015 to 05/31/2018 at Mayo Clinic, Florida. We included patients who were part of the "fast track" protocol at our institution as they would receive neuromuscular blockade antagonism and be extubated in the operating room at the conclusion of the case. "Fast track" liver transplantation is defined as extubating in the operating room, bypassing the ICU, and going directly from the post-anesthesia care unit to the surgical ward. ${ }^{6}$ We collected the patient's baseline characteristics, intraoperative variables, and postoperative variables using our institution's medical record system (Epic, Verona, WI). Baseline characteristics included age, sex, weight, body mass index (BMI), physiologic model for end-stage liver disease (MELD) score on the day of surgery, and liver graft types, including donation after circulatory death (DCD) or donation after brain death (DBD). Both preoperative and immediate postoperative laboratory variables were collected, which included international normalized ratio (INR), aPTT, and creatinine. We also collected blood products transfused in the postoperative day (POD) 0 to day 1, POD 0-1 respiratory complication, POD 0 PONV status, POD 0-1 need for re-operation for bleeding, POD 0-7 organ rejection, and the length of stay. PONV status is defined as if the patient needed rescue antiemetics. POD 0-7 organ rejection was confirmed by biopsy in the first-week post-transplant.

Induction of anesthesia was at the anesthesiologist's discretion and performed using 1 to $2 \mathrm{ug} / \mathrm{kg}$ fentanyl, $0.6 \mathrm{mg} / \mathrm{kg}$ rocuronium or $1 \mathrm{mg} / \mathrm{kg}$ succinylcholine, and 2 to $2.5 \mathrm{mg} / \mathrm{kg}$ propofol. Intraoperatively patient received rocuronium based on subjective evaluation with a peripheral nerve stimulator (PNS); maintenance of anesthetic with sevoflurane. All patients were kept normothermic with forced-air warming devices, warming mattresses, and a fluid warmer. Intraoperative coagulation labs included platelet count (Plts), PT/INR, 
aPTT, fibrinogen, and thromboelastography (TEG) were collected post-induction, 10 minutes before and after the anhepatic phase, 5 minutes before and after the reperfusion phase. Additional labs and TEG were collected at the discretion of the attending anesthesiologist. Patients received various blood products based on the lab results with a goal of the following: $\mathrm{Hgb} 9$ to $10 \mathrm{mg} / \mathrm{dl}$, INR 1.5 to 2.0, fibrinogen above 180, Plts around 100, 000 .

The anesthesiologist and the transplant surgeon made the decision to "fast-track" based on their clinical judgment rather than a defined clinical protocol. At the time of emergence, all patients received either sugammadex $(2-4 \mathrm{mg} / \mathrm{kg})$ or neostigmine $(0.03-0.07 \mathrm{mg} / \mathrm{kg})$ based on values obtained from the PNS. All patients were extubated after the NMBA antagonist was administered, and the anesthesia team felt neuromuscular function had been restored based on values from a PNS.

The primary outcomes were POD 0-1 bleeding events (re-operation for bleeding, packed red blood cell (PRBC) transfusion), and POD 0 values of aPTT and INR. Secondary outcomes were POD 0-1 respiratory complication, POD 0 PONV, POD 0-7 organ rejection, and length of stay.

Continuous variables were summarized as mean (standard deviation) or median (range), while categorical variables were reported as frequency (percentage). Continuous baseline and preoperative variables were compared between the standard and sugammadex groups using the Wilcoxon rank-sum test, and categorical variables were compared using the Chi-squared test. All tests were two-sided, with an alpha level set at 0.05 for statistical significance. The difference and corresponding $95 \%$ confidence intervals (CI) in the continuous postoperative outcome variables between the two groups were estimated using linear regression models, and odds ratios and $95 \% \mathrm{CI}$ in dichotomous outcomes were estimated using logistic regression models.

\section{Results}

A total of 241 patients were included in the study, with 135 receiving neostigmine and 106 receiving sugammadex for the reversal of neuromuscular blockade. There was no significant difference between the MELD score for patients receiving sugammadex or neostigmine $(13.7 \pm 6.9$ vs. $12.3 \pm 7.5, \mathrm{p}=0.10)$, respectively. There was also no difference in the donor process (DCD vs. $\mathrm{DBD})$ between the two groups $(\mathrm{P}=0.13)$ (Table 1$)$.

After the completion of liver transplantation, immediate POD 0-1 bleeding requiring transfusion occurred in $20 \%$ of patients in the neostigmine group vs $10.4 \%$ in the sugammadex group (difference $0.46 \%[95 \% \mathrm{Cl}$, $0.22 \%$ to $0.98 \%]$ ). POD $0-1$ reoperation for bleeding occurred in $1.5 \%$ of patients in neostigmine group vs $0 \%$ in sugammadex group (difference $0.25 \%$ [95\% Cl, $0.01 \%$ to $5.35 \%]$ ). POD 0 mean INR was $2.0 \pm 0.4$ in both groups. POD 0 mean aPTT was $45.5 \pm 7.9$ in neostigmine group vs $49.3 \pm 9.0$ in sugammadex group (difference $3.8 \%$ [ $95 \% \mathrm{Cl}, 1.65 \%$ to $5.94 \%]$ ). Among secondary outcomes, PONV POD 0 occurred in $32.6 \%$ of patients in neostigmine group vs $29.2 \%$ in sugammadex group (difference $0.86 \%$ [ $95 \% \mathrm{Cl}, 0.49 \%$ to $1.49 \%$ ]). Acute organ rejection occurred in $0.7 \%$ patients in neostigmine group vs $1.9 \%$ in sugammadex group (difference $2.58 \%$ [ $95 \% \mathrm{Cl}, 0.23 \%$ to $28.81 \%$ ], $p=0.58$ ). Length of stay is similar with mean $7.0 \pm 4.6$ ) days in neostigmine group vs $7.8 \pm 5.7$ ) days in sugammadex group (difference 0.87 [95\% Cl, -0.44 to 2.17]) (Table 2). The mean differences and $95 \%$ CI displayed were demonstrated in Figure 1. The difference in POD 0 PTT was significantly different between the two groups.

\section{Discussion}

In our review of 241 patients undergoing liver transplantation, we found no statistically significant difference in postoperative bleeding outcomes in patients reversed with sugammadex vs. neostigmine. Compared with neostigmine, the use of sugammadex had slightly prolonged aPTT at the immediate postoperative period; however, it was associated with less risk of postoperative red blood cell transfusion and re-operation. In the secondary outcomes, acute organ rejection occurred slightly higher in the sugammadex group; however, this slight increase was not statistically significant. The sugammadex group had less PONV. Both groups had similar LOS.

Postoperative hemorrhage is one of the broad spectra of liver transplantation complications. Mueller et al. suggested that the most important risk factors for early postoperative bleeding are severe coagulopathy and 
thrombocytopenia. ${ }^{3}$ Everson et al. proposed that the most significant bleeding risk is in the setting of both severe coagulopathy and portal hypertension..$^{7}$ Abnormal coagulation reflected by prolongation of aPTT and $\mathrm{PT}$ are common laboratory findings in these patients. Patients with cirrhosis have been described as having a rebalanced hemostatic system due to a concurrent reduction in pro- and anti-hemostatic. systems ${ }^{8,}{ }^{9}$ However, the hemostatic balance in these patients is more fragile compared to healthy individuals. Both diffuse bleeding and thrombosis can occur during and after liver transplantation. After liver transplantation, it is presumed that the hemostatic system normalizes as the liver synthetic function is restored.

Clinical trials in healthy subject and surgical patients reported that sugammadex had transient aPTT and PTT prolongations. ${ }^{4,10}$ Based on the information supplemented by the Food and Drug Administration (FDA), healthy volunteers had increased aPTT and PT/INR up to 1 hour with administration doses up to $16 \mathrm{mg} / \mathrm{kg}$ sugammadex. ${ }^{11}$ Dirkmann et al. suggested the effect on coagulation may be explained by the cyclodextrin molecule binding the phospholipid, and the anticoagulant effects were likely an in vitro artifact observed in commercial phospholipid-dependent assays such as the aPTT. ${ }^{12}$ Other studies found that sugammadex does not affect platelet or factor Xa function. ${ }^{13,14}$ However, the sugammadex effect on postoperative bleeding is still controversial. Tas et al. reported that sugammadex was associated with a higher amount of postoperative bleeding than neostigmine after septoplasty in otherwise healthy patients. ${ }^{15}$

On the contrary, Raft et al. investigated postoperative bleeding in high-risk cancer patients, and they found sugammadex was not associated with increased postoperative bleeding. ${ }^{16}$ Moon et al . reported that sugammadex is not associated with increased bleeding tendency or morbidity in healthy patients undergoing living-donor hepatectomy. ${ }^{17}$ Our study had similar results as sugammadex was not associated with an increased bleeding profile. In fact, there was no instance of re-operation in the sugammadex group. To the best of our knowledge, this is the first effort to examine the bleeding risk of sugammadex in deceased donor liver transplantation.

In the secondary outcomes, the sugammadex group had slightly higher acute organ rejection $(0.7 \%$ patients in the neostigmine group vs. $1.9 \%$ in the sugammadex group). However, both groups had a much lower acute organ rejection rate than large cohort studies. (27\% in the Adult to Adult Living Donor Liver Transplantation cohort and $15.6 \%$ in the Scientific Registry of Transplant Recipients cohort. ${ }^{18}$ Sugammadex creates a steroid complex with the aminosteroid neuromuscular blocking agents, and it has been shown to reduce other drugs' efficacy by encapsulating drugs with similar structures such as oral contraceptive pills. ${ }^{11}$ Corticosteroids are a crucial component of immunosuppression therapy in liver transplantation and are administered during the peri-transplant period to prevent acute organ rejection. Rezonja et al. found the concomitant administration of high dose dexamethasone diminished the efficiency of sugammadex in the in vitro experiment. ${ }^{19}$ However, Rezonja et al. also found that dexamethasone does not diminish sugammadex reversal of neuromuscular blockade in a clinical study. ${ }^{20}$ Furthermore, Arslantas et al. found no difference in the risk of adverse effects on short-term graft function in 42 patients who underwent kidney transplantation. ${ }^{21}$

Our study also showed that the sugammadex group had less PONV, which is consistent with previous work. ${ }^{3}$ Regarding hospital length of stay, the two groups had small differences: 7.0 days in the neostigmine group and 7.8 days in the sugammadex group. In a large cohort study, patients reversed with sugammadex had earlier first postop bowel movement compare to patients reversed with neostigmine ${ }^{22}$, which potentially could reduce the hospital length of stay.

There are several limitations to our study. First, the retrospective design of the study is certainly a limiting factor. Additionally, this data represents outcomes from a single-center, although our institution performs roughly 150 liver transplantations a year, and there were no significant differences in any of our demographic variables. Third, temporal factors exist as the neostigmine group was transplanted from 2015 to 2017, versus the sugammadex group which was transplanted from 2017 to 2018. Surgical techniques and surgeon experiences may have improved with time and could confound our results. Nevertheless, our study is the first study to evaluate the sugammadex effect on postoperative bleeding risk in the adult orthotopic liver transplantation population. 


\section{Conclusion}

Our retrospective study results demonstrate that sugammadex is not associated with an increase in postoperative bleeding events, had less PONV, did not affect the organ rejection rate, and did not affect hospital length of stay. We provide incremental evidence sugammadex can be considered as an alternative to antagonize neuromuscular blockade following orthotopic liver transplantation.

\section{Author Contribution :}

1. Hong Liang: Drafting and revision of the article, Data collection.

2. Ryan Chadha: Concept and study design, critical revision of article

3. J. Ross Renew: Concept and study design, critical revision of article. Approval of article.

4. Launia J White: Data analysis and interpretation and statistics.

\section{Data Sharing:}

The data that support the findings of this study are available from the corresponding author upon reasonable request.

\section{References:}

1. OPTN/SRTR 2018 annual data report: Introduction. Am J Transplant. 2020;20 Suppl s1(s1):11-9.

2. Brull SJ, Naguib M. Selective reversal of muscle relaxation in general anesthesia: focus on sugammadex. Drug Des Devel Ther. 2009;3:119-29

3. Hristovska A-M, Duch P, Allingstrup M, Afshari A. Efficacy and safety of sugammadex versus neostigmine in reversing neuromuscular blockade in adults. Cochrane Database Syst Rev. 2017;8:CD012763.

4. De Kam P-J, Grobara P, Prohn M, Höppener F, Kluft C, Burggraaf J, et al. Effects of sugammadex on activated partial thromboplastin time and prothrombin time in healthy subjects. Int J Clin Pharmacol Ther. 2014;52(3):227-36.

5. Mueller A, Platz K, Kremer B. Early postoperative complications following liver transplantation. Best Pract Res Clin Gastroenterol. 2004;18(5):881-900.

6. Taner CB, Willingham DL, Bulatao IG, Shine TS, Peiris P, Torp KD, et al. Is a mandatory intensive care unit stay needed after liver transplantation? Feasibility of fast-tracking to the surgical ward after liver transplantation. Liver Transpl. 2012;18(3):361-9.

7. Everson GT. A hepatologist's perspective on the management of coagulation disorders before liver tranplantation. Liver Transpl Surg. 1997;3(6):646-52.

8. Roberts LN, Patel RK, Arya R. Haemostasis and thrombosis in liver disease. Br J Haematol. 2010;148(4):507-21.

9. Tripodi A, Mannucci PM. The coagulopathy of chronic liver disease. N Engl J Med. 2011;365(2):147-56.

10. Rahe-Meyer N, Fennema H, Schulman S, Klimscha W, Przemeck M, Blobner M, et al. Effect of reversal of neuromuscular blockade with sugammadex versus usual care on bleeding risk in a randomized study of surgical patients. Anesthesiology. 2014;121(5):969-77.

11. Summary of product characteristics, Bridion. 2015. Fda.gov. [cited 2021 Jan 18]. Available from: https://www.accessdata.fda.gov/drugsatfda_docs/label/2015/022225lbl.pdf

12. Dirkmann D, Britten MW, Pauling H, Weidle J, Volbracht L, Görlinger K, et al. Anticoagulant effect of Sugammadex: Just an in vitro artifact. Anesthesiology. 2016;124(6):1277-85.

13. De Kam P-J, El Galta R, Kruithof AC, Fennema H, van Lierop M-J, Mihara K, et al. No clinically relevant interaction between sugammadex and aspirin on platelet aggregation and coagulation parameters. Int J Clin Pharmacol Ther. 2013;51(12):976-85.

14. De Kam P-J, Kruithof AC, van Lierop M-J, Moerland M, Dennie J, Troyer MD, et al. Lack of a clinically relevant effect of sugammadex on anti-Xa activity or activated partial thromboplastin time following pretreatment with either unfractionated or low-molecular-weight heparin in healthy subjects. Int J Clin Pharmacol Ther. 2014;52(8):631-41.

15. Taş N, Korkmaz H, Yağan Ö, Korkmaz M. Effect of sugammadex on postoperative bleeding and coagulation parameters after septoplasty: A randomized prospective study. Med Sci Monit. 2015;21:2382-6. 
16. Raft J, Betala Belinga JF, G J, Desandes E, Longrois D, Meistelman C. Clinical evaluation of postsurgical bleeding after a sugammadex injection. Ann Fr Anesth Reanim 2011; 30:714

17. Moon Y-J, Kim S-H, Kim J-W, Lee Y-K, Jun I-G, Hwang G-S. Comparison of postoperative coagulation profiles and outcome for sugammadex versus pyridostigmine in 992 living donors after living-donor hepatectomy. Medicine (Baltimore). 2018;97(11):e0129.

18. Levitsky J, Goldberg D, Smith AR, Mansfield SA, Gillespie BW, Merion RM, et al. Acute rejection increases risk of graft failure and death in recent liver transplant recipients. Clin Gastroenterol Hepatol. 2017;15(4):584-593.e2.

19. Rezonja K, Sostaric M, Vidmar G, Mars T. Dexamethasone produces dose-dependent inhibition of sugammadex reversal in in vitro innervated primary human muscle cells. Anesth Analg. 2014;118(4):75563.

20. Rezonja K, Mars T, Jerin A, Kozelj G, Pozar-Lukanovic N, Sostaric M. Dexamethasone does not diminish sugammadex reversal of neuromuscular block - clinical study in surgical patients undergoing general anesthesia. BMC Anesthesiol. 2016;16(1):101

21. Arslantas R, Cevik BE. Retrospective investigation of grafted kidney function after reversal of neuromuscular blockade using neostigmine or sugammadex. Transplant Proc. 2019;51(7):2265-7.

22. Deljou A, Schroeder DR, Ballinger BA, Sprung J, Weingarten TN. Effects of sugammadex on time of first postoperative bowel movement: A retrospective analysis. Mayo Clin Proc Innov Qual Outcomes. 2019;3(3):294-301.

Tables

Table 1. Patient characteristics and comparison of preoperative variables.

Episodes of Rejection (POD \#1-

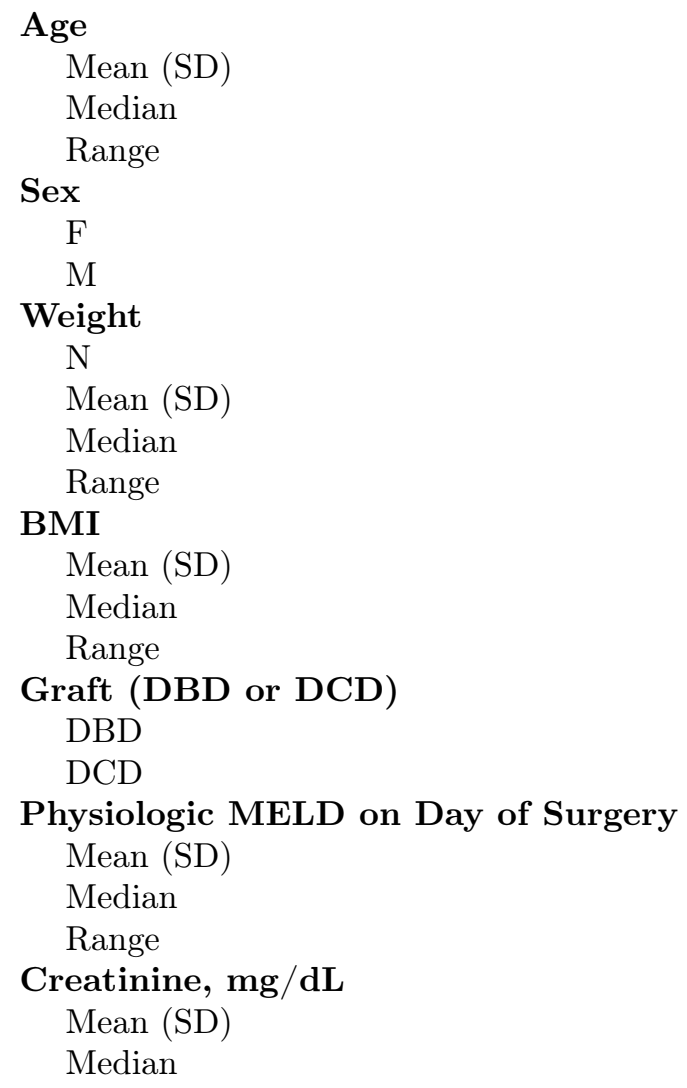




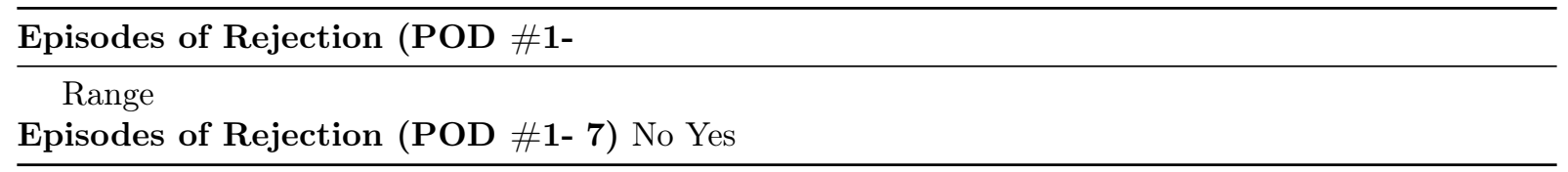

Table 2. Differences and $95 \% \mathrm{CI}$ in outcome variables between groups

\begin{tabular}{|c|c|c|c|}
\hline & Standard $(\mathrm{N}=135)$ & Sugammadex $(N=106)$ & $\begin{array}{l}\text { Mean Difference }(95 \% \\
\text { CI) }\end{array}$ \\
\hline \multicolumn{4}{|c|}{$\begin{array}{l}\text { POD\# } 1 \text { PRBC } \\
\text { transfusion }\end{array}$} \\
\hline No & $108(80.0 \%)$ & $95(89.6 \%)$ & $0.46(0.22,0.98)$ \\
\hline Yes & $27(20.0 \%)$ & $11(10.4 \%)$ & \\
\hline \multicolumn{4}{|l|}{ POD \#0-1 } \\
\hline \multicolumn{4}{|c|}{$\begin{array}{l}\text { PRBC Transfu- } \\
\text { sion (units) }\end{array}$} \\
\hline Mean (SD) & $0.4(0.9)$ & $0.2(0.5)$ & $-0.23(-0.43,-0.04)$ \\
\hline Median & 0.0 & 0.0 & \\
\hline \multicolumn{4}{|l|}{ POD \#0-1 } \\
\hline \multicolumn{4}{|c|}{ Re-operation for } \\
\hline \multicolumn{4}{|c|}{ Bleeding } \\
\hline No & $133(98.5 \%)$ & $106(100.0 \%)$ & $0.25(0.01,5.35)$ \\
\hline Yes & $2(1.5 \%)$ & $0(0.0 \%)$ & \\
\hline Median & 1.8 & 1.9 & \\
\hline \multicolumn{4}{|c|}{ POD $\# 0$ aPTT } \\
\hline Mean (SD) & $45.5(7.9)$ & $49.3(9.0)$ & $3.80(1.65,5.94)$ \\
\hline Median & 44.1 & 47.8 & \\
\hline \multicolumn{4}{|l|}{ LOS } \\
\hline Mean (SD) & $7.0(4.6)$ & $7.8(5.7)$ & $0.87(-0.44,2.17)$ \\
\hline Median & 5.0 & 6.0 & \\
\hline \multicolumn{4}{|c|}{ POD \#0 PONV } \\
\hline No & $91(67.4 \%)$ & $75(70.8 \%)$ & $0.86(0.49,1.49)$ \\
\hline Yes & $44(32.6 \%)$ & $31(29.2 \%)$ & \\
\hline \multicolumn{4}{|l|}{ POD \#1-7 } \\
\hline \multicolumn{4}{|c|}{ Organ Rejection } \\
\hline No & $134(99.3 \%)$ & $104(98.1 \%)$ & $2.58(0.23,28.81)$ \\
\hline Yes & $1(0.7 \%)$ & $2(1.9 \%)$ & \\
\hline
\end{tabular}

$\mathrm{POD}=$ Postoperative day $\mathrm{SD}=$ Standard Deviation; INR $=$ International normalized ratio; aPTT $=$ Activated thromboplastin time; $\mathrm{PRBC}=$ Packed red blood cell; $\mathrm{LOS}=$ Length of stay; PONV = Postoperative nausea and vomiting.

\section{Figure legend}

Figure 1. Forest plot displaying the mean differences in outcome variables between groups (The mean differences and 95\% CI displayed were the estimated coefficients and 95\%CI from linear regression models for continuous outcomes and logistic regression models for categorical outcomes respectively. When the $95 \% \mathrm{CI}$ does not include 0 , it means the difference in that outcome was significantly different between the two groups) 


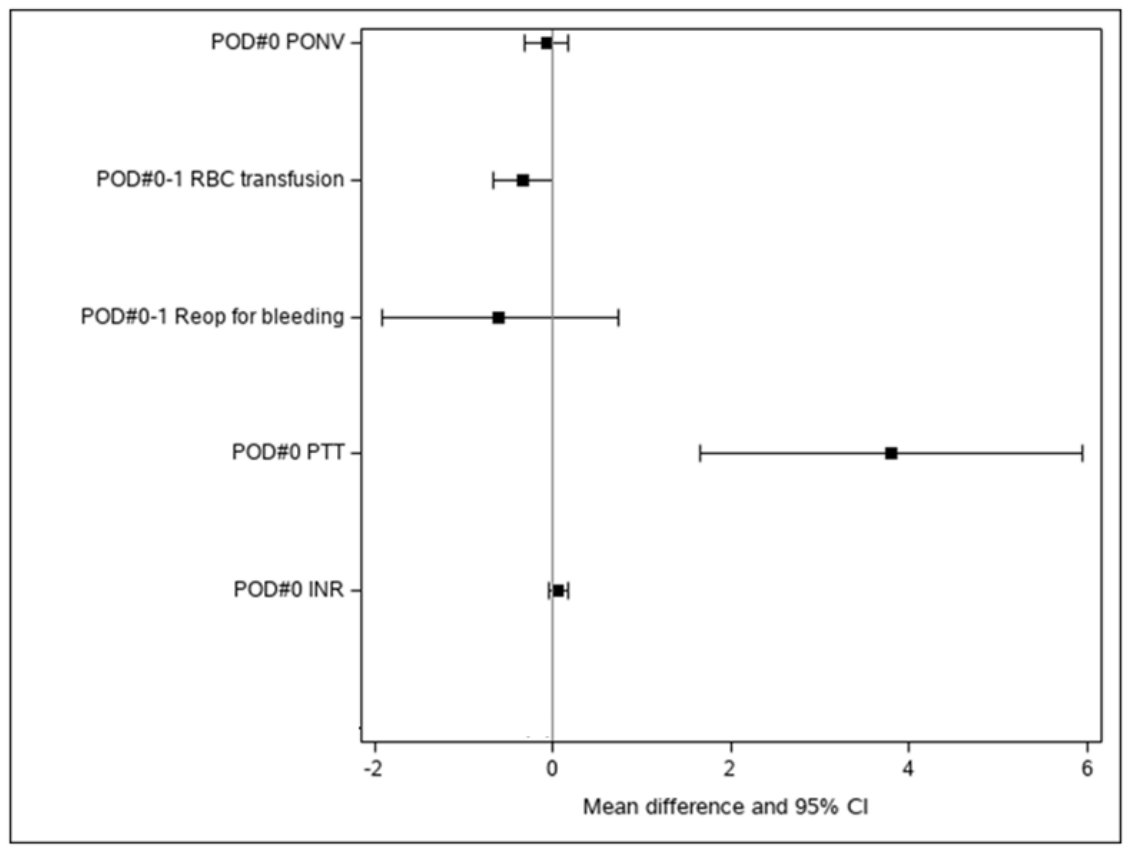

POD\#0 PONV, POD 0-1 RBC transfusion, POD 0-1 reoperation for bleeding all favor sugammadex. POD0 PTT favors neostigmine. $\mathrm{POD}=$ postoperative day; $\mathrm{PONV}=$ postoperative nausea and vomiting; $\mathrm{RBC}=$ red blood cell; $\mathrm{PTT}=$ partial thromboplastin time; $\mathrm{INR}=$ International normalized ratio. 